\title{
Brain Computer Interface System for Mind Controlled Robot using Bluetooth
}

\author{
Siliveru Ramesh \\ M.Tech Student \\ Dept of ECE \\ Vardhaman College of \\ Engineering \\ Shamshabad, Hyderabad, India
}

\author{
M.Gopi Krishna \\ Assistant Professor \\ Dept of ECE \\ Vardhaman College of \\ Engineering \\ Shamshabad, Hyderabad, India
}

\author{
Madhu Nakirekanti \\ Assistant Professor \\ Dept of ECE \\ Vardhaman College of \\ Engineering \\ Shamshabad, Hyderabad, India
}

\begin{abstract}
This paper describes the Mind Controlled Robot based on Brain Computer Interface (BCI) using LabVIEW to analysis the brain waves. BCIs are systems that may bypass typical channels of communication (i.e., muscles and thoughts) to supply direct communication and management between the human brain and physical devices by translating different patterns of brain activity into commands in real time. With these commands a mobile robot can be controlled. The intention of the project work is to develop a mechanism that may assist the disabled folks in their everyday life to do some work freelance on others. Here, they tend to are analyzing the brain wave signals. Human brain consists of innumerable interconnected neurons. The patterns of interaction between these neurons are delineating as thoughts and emotional states. In step with the human thoughts, this pattern are going to be dynamical that successively manufacture totally different electrical waves [1].
\end{abstract}

\section{Keywords}

EEG sensor, Brain Computer Interface (BCI), communication module, relay Driver.

\section{INTRODUCTION}

The fundamental plan of $\mathrm{BCI}$ is to translate user created patterns of brain activity into corresponding commands. A typical BCI consists of signal acquisition and signal process including preprocess, feature extraction and classification. Although some BCI systems don't embrace all elements cluster 2 or 3 elements into one rule, most systems will be conceptually divided into signal acquisition, preprocess, feature extraction, and classification [2]. The brain signals that are widely used to develop EEG-based BCIs embrace P300 potentials, that are a positive potential deflection on the continuing brain activity at a latency of roughly $300 \mathrm{~ms}$ once the random incidence of a desired target stimulation from no target stimuli (the stimuli will be in visual, auditory, [3] or tactile modality SSVEP, that are visually induced by a stimulation modulated at a set frequency and occur as a rise in graphical record activity at the stimulation frequency and the event-related American state synchronization (ERD) and event-related synchronization (ERS), which are induced by performing mental tasks, such as motor imagery, mental arithmetic, or mental rotation [4].

\section{DESIGN OF SYSTEM}

This paper is enforced in an efficient means it has a tendency to divide brain-controlled mobile robots into 2 classes consistent with their operational modes. One class is termed "direct management by the BCI," which implies that the BCI interprets graphical record signals into motion commands to regulate robots directly United Nations agency 1st developed a brain-controlled robotic chair whose left or right turning movements are directly controlled by corresponding motion commands translated from user brain signals whereas imagining left or right limb movements, and tested this technique in real-world things [5]. The robotic platform is illustrated also used a BCI based on motor imagery to build a brain-controlled mobile robot, as illustrated which might perform 3 motion commands together with turning left and right and going forward, and valid this automaton during a planet.

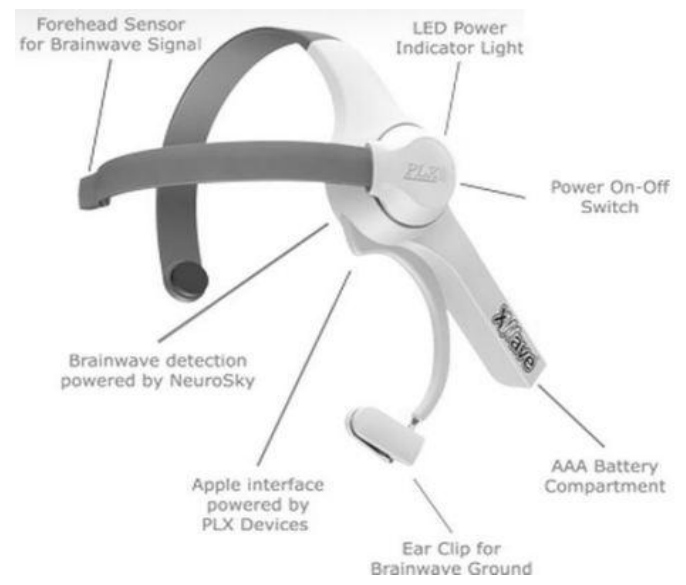

Fig 1: Mindwave Headset provided by Neurosky

In figure 1, the Mindwave Headset which is provided by Neurosky Technologies and those signals will be transferred by using Bluetooth which is there in the Mindwave headset, for this Mindwave headset need to give power using an AAA battery. The Mindwave headset comes with Power switch, a sensor tip, flexible ear arm and a ground connection Ear clip. In this Headset they use Non-invasive sensor that won't cause any pain to the User who were the headset. After inserting an AAA battery switch on the Mindwave headset using the power switch the LED indicator will blink and if the Red color light not blinking the headset is powered on but not connected to with the computer's Bluetooth. If the Blue color not blinking that means the headset is powered on and connected. If the red or blue color blinks it shows that the Battery getting low.

As shown in figure 2 the Data transmitted by the Mindwave headset will be received by the Computer's Bluetooth receiver. And then all these data will be analyzed by the Level Analysis platform. The Level Analysis platform will extract 
the raw data using the LabVIEW. In the LabVIEW the data will be received from the port pin which they are giving the same port number for the Bluetooth receiver and LabVIEW in the back panel.

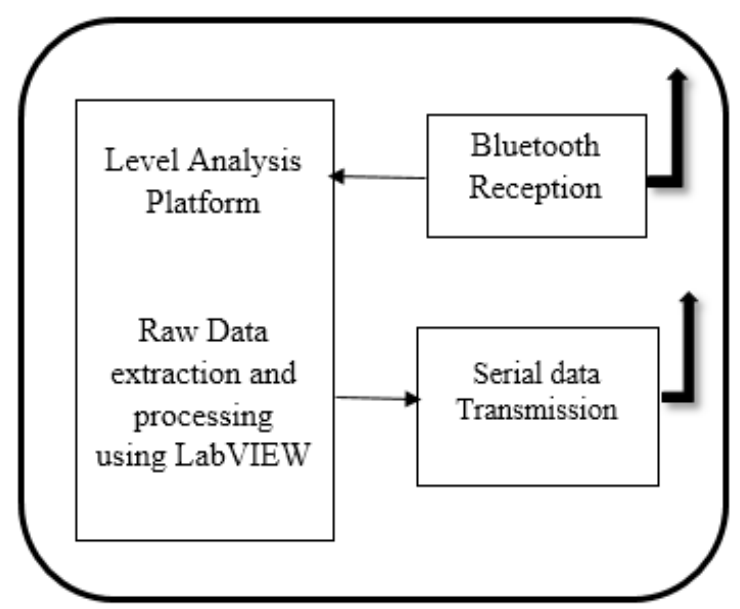

Fig 2: DATA Processing Unit

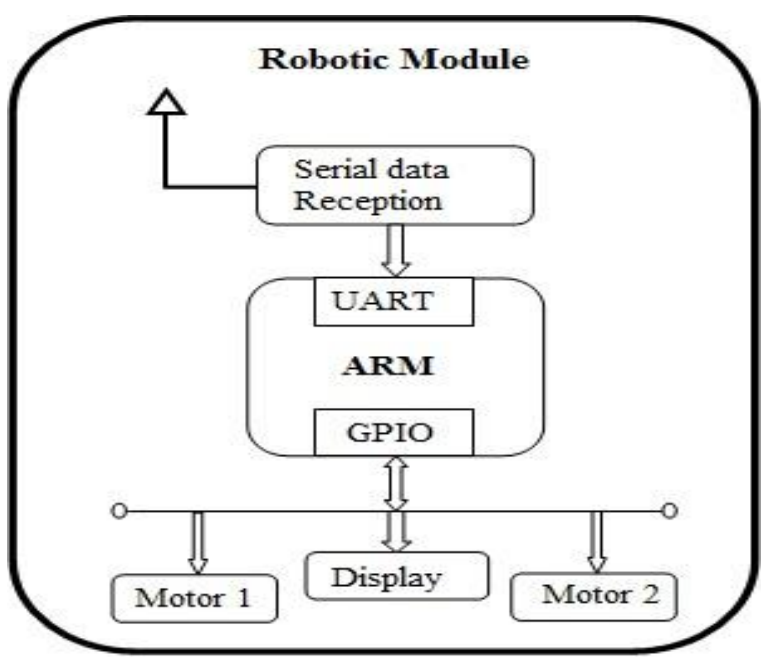

Fig 3: Robotic Module

After the analysis of this data, this data will be sent to the robot module using serial data transmission i.e. using XBee. As shown in figure 3 in the robot module there will be an $\mathrm{XBee}$ receiver will receive the data which is transmitted by the XBee transmitter. According to the data received by the XBee the ARM processor will give the directions to the motors which are connected with a relay and a driver circuit. And all this information will be displayed on the LCD display.

\section{SYSTEM HARDWARE}

\subsection{ARM Processor}

The ARM processor is 32-bit embedded reduced instruction set computer microprocessor. The ARM7 processor desires terribly low power, high performance and little size. Here during this paper they victimization ARM7 method or an ARM processor can receive the signals from the Bluetooth receiver and it'll process the signals and it'll provide the signals to the driving force Circuit and in line with that signals received from the processor, in line with the signals received driver circuit the motors can rotate forward, clockwise and anti-clockwise direction. Here ARM processor can wait till the signals received from the Mindwave headset and when receiving the signals it'll moves the robots. The driving force circuit can connected to the port one of the processor.

\subsection{Wireless communication}

The XBee and XBee-PRO OEM RF Modules were designed to satisfy IEEE 802.15.4 standards and support the distinctive wants of low-priced, low-power wireless detector networks [6]. The modules need stripped-down power and supply reliable delivery of knowledge between devices. The modules operate inside the belief a pair of. $4 \mathrm{GHz}$ waveband. Here they tend to are victimization XBee to attach automaton with the pc wirelessly through a logic-level asynchronous port. Through its port, the module will communicate with any logic and voltage compatible UART [7]. The pc can remodel the information that is analyzed by victimization the amount analyzer unit victimization MAT workplace. The information that is transmitted by the XBee module from the pc are going to be received by the $\mathrm{XBee}$ receiver that is connected to the ARM processor.

\subsection{EEG Signals}

EEG signals will be collected with electrodes that area unit placed on the surface of the scalp. The foremost wide used electrodes area unit silver/silver chloride $(\mathrm{Ag} / \mathrm{AgCl})$ as a result of they need low price, low contact electric resistance, and comparatively sensible stability. What is more, there are a unit rather mature commercial acquisition systems together with the electronic equipment and graphical record cap with integrated $\mathrm{Ag} / \mathrm{AgCl}$ electrodes that are with success applied in research and clinical diagnosing. However, exploitation $\mathrm{Ag} / \mathrm{AgCl}$ electrodes need removing outer skin layer and filling gel between electrodes and scalp (and so, this sort of electrodes is additionally known as "wet" electrodes). These operations take lasting and area unit uncomfortable to users. To deal with these limitations of "wet" electrodes, some researchers are exploring "dry" electrodes that ought not to use gel and skin cleanup [8-11]. The most disadvantages of existing dry electrodes is that the no heritable graphical record signals area unit worse than those uninhabitable with standard electrodes as a result of the rise of contact electric resistance [12]. Some firms are commercializing acquisition systems supported dry electrodes $[13,14]$. Here they tend to area unit exploitation Neurosky Mindwave telephone receiver. However, they're not nonetheless mature, and a few researchers have doubts concerning what physiological signals these systems really acquire [15]. Therefore, until now, all brain-controlled wheelchairs adopt "wet" electrodes to gather brain signals.

\subsection{NeuroSky Technology}

\subsubsection{Brainwaves}

The last century of neurobiology analysis has greatly hyperbolic our information regarding the brain and significantly, the electrical signals emitted by neurons firing within the brain. The patterns and frequencies of those electrical signals may be measured by inserting a detector on the scalp. The Mind Tools line of receiver product contains NeuroSky ThinkGear technology that quantifies the analog electrical signals, ordinarily remarked as brainwaves, and exercises them into digital signals. The ThinkGear technology then makes those computations and signals out there to games and applications. The Table I provides a general precise of a number of the commonly- known frequencies that tend to be generated by differing kinds of activity within the brain [16]. 


\subsubsection{ThinkGear}

ThinkGear is that the technology within each NeuroSky product or partner product that empowers a tool to interface with the wearers' brainwaves [16]. It includes the detector that touches the forehead, the contact and reference points settled within the ear clip, and also the on-board chip that processes all of the info. Each the raw brainwaves and also the eSense Meters square measure computed on the ThinkGear chip.

\subsection{Design Flow}

The flow diagram of Mindwave Controlled Robot unit is shown in figure 4 . It shows all the step by step functions of robot, how it will be controlled by using brainwave signals. After Switching on the Mindwave headset and the Robot kit, the processor will initialize and the headset will starts sensing the neurons signals and after sensing the signals it will transfer them to through the Bluetooth and the acquisition module will receive the signals in the processor and in the processor the EEG signals comparison will be done if it is yes then the robot will move according to the signals or else it will go to the relay circuit and robot movement will be there and the process will be stopped.

\section{EXPERIMENTAL STUDY}

This system uses ARM processor as a core and wireless sensor network communication as a transmission medium for the data transfer. This system consists of three important sections. One is Brainwave headset provided by Neurosky, the other one is Robot and the Monitoring section in PC which is using LabVIEW. This Headset and the PC will be interlinked with the help of Bluetooth wireless communication and the PC and Robot interlink with the Zigbee wireless communication. To connect Headset with PC they need to

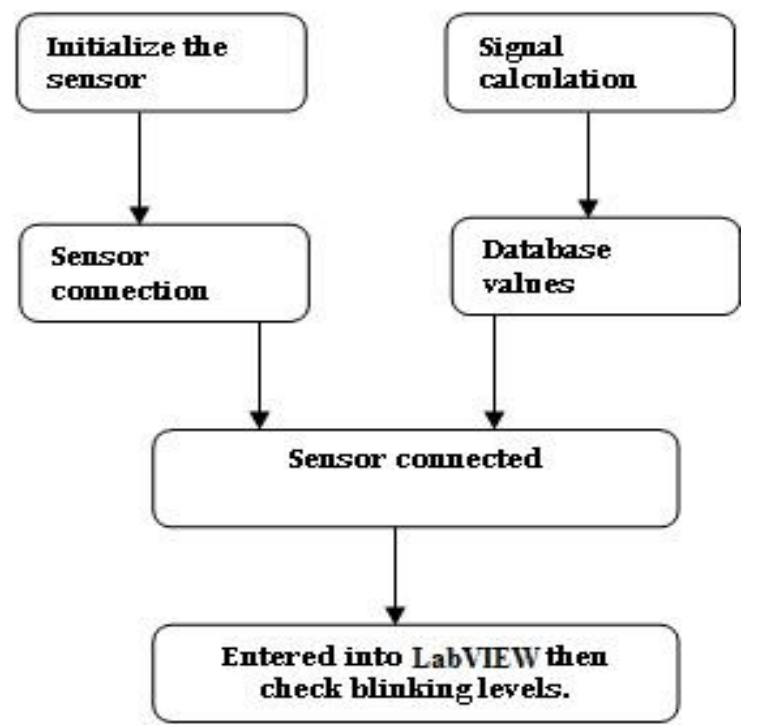

Fig 4: Design Flow

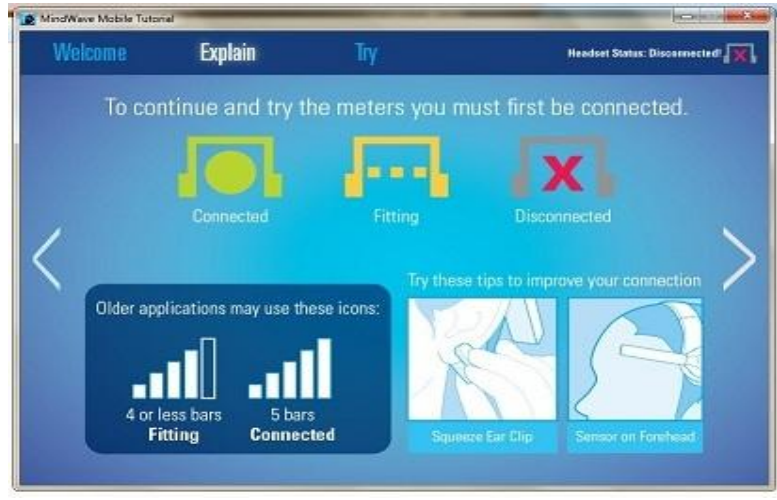

Fig 5: Neurosky Mindwave core design

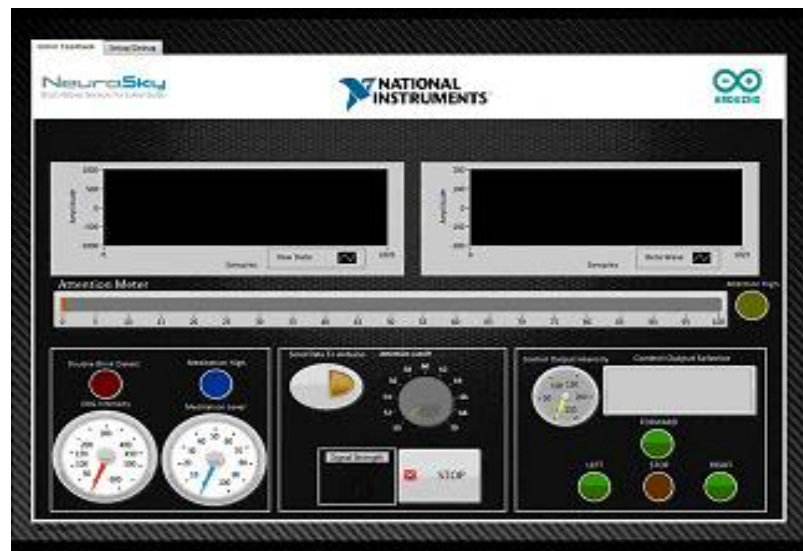

Fig 6: LabVIEW front Panel

install the Neurosky software on PC and after that switch on the Headset and try to connect with the headset to the PC by opening the NeuroSky software, after opening the software it show like in the figure 5, and connect with PC. Now open the LabVIEW and run the program and it shows like in figure 6 and monitor the brain waves. These signal processing is done on LabVIEW on raw EEG Waveform bring out Attention Level and Eye blinks and these two parameters are used to control Robot movements. Power spectrum of Beta wave

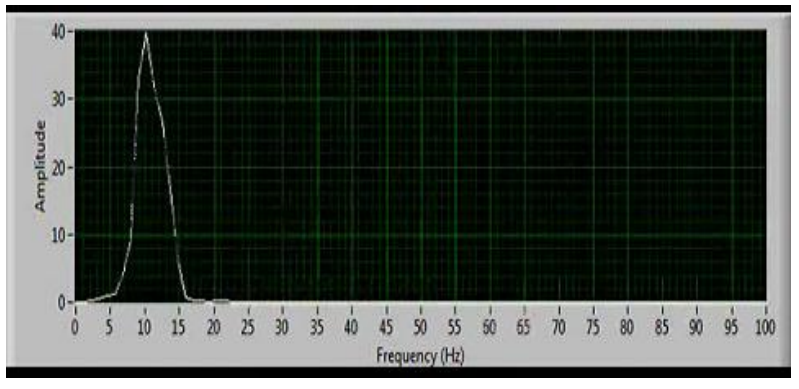

Fig 7: Attention Level Graph 


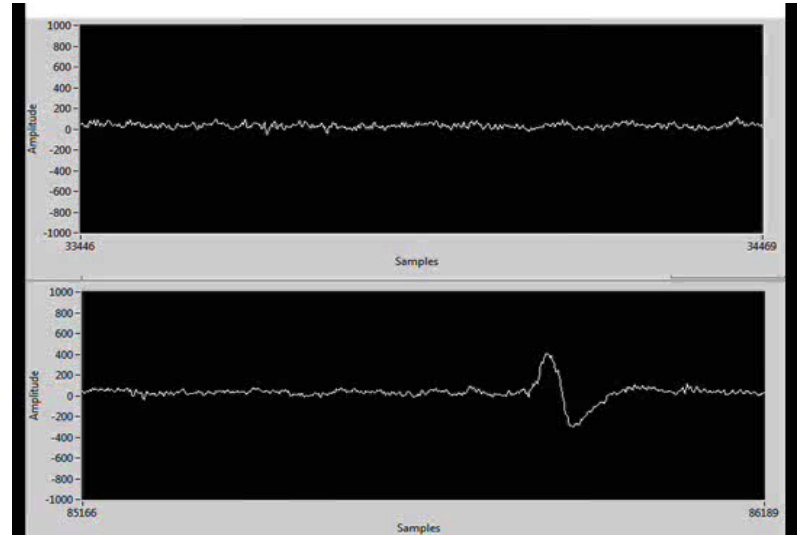

Fig 8: Blink Level Graph

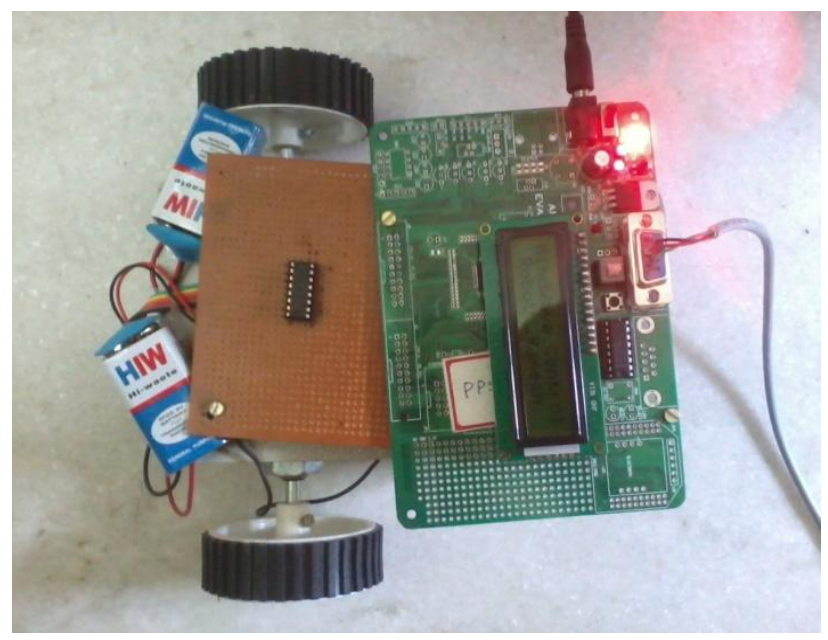

Fig 9: Final Prototype

extracted through simple processing techniques gives Attention level which is shown in figure 7 and the eye blink is shown in figure 8. These two attention level and blink is used to select the direction of robot. If Single blink is detected then robot moves clockwise and if Double blink is detected then robot will move anti-clockwise direction and based on attention level the robot will forward direction.

\section{CONCLUSION}

The analysis and development of brain-controlled mobile robots have received an excellent deal of attention as a result of they'll facilitate bring quality back to folks with devastating contractile organ disorders and therefore improve their quality of life. During this paper, they tend to confer a comprehensive up-to-date review of the whole systems, key techniques, and analysis problems with brain-controlled mobile robots.

\section{REFERENCES}

[1] Luzheng Bi, Xin-An Fan, Yili Liu "EEG-Based Braincontrolled Mobile Robots: A survey", Human-Machine Systems, IEEE Transactions on (Volume: 43, Issue: 2), pp. 161-176, Mar 2013.

[2] Kale Swapnil T, Mahajan Sadanand P, Rakshe Balu G, Prof. N.K.Bhandari "Robot Navigation control through EEG Based Signals" International Journal Of Engineering And Computer Science ISSN:2319-7242 Volume 3 Issue 3 March-2014 Page No. 5105-5108.

[3] Priyanka.M Manju Paarkavi.R Dhanasekhar.S "An Intelligent Acoustic Communication System for Aphasia Forbearings" International Conference on Signal
Processing, Embedded System and Communication Technologies and their applications for Sustainable and Renewable Energy (ICSECSRE '14), Vol. 3, Special Issue 3, April 2014.

[4] John Jonides, Patricia A. Reuter-Lorenz, Edward E.Smith, Edward Awh, Lisa L.Barnes, Maxwell drain, Jennifer Glass, Erick J.Lauber, Andrea L.Patalano, Eric H.Schumacher, "Verbal and Spatial Working Memory in Humans" The Psychology of Learning and Motivation, Vol.35.

[5] I.I. Goncharova, D.J. McFarland, T.M. Vaughan, J.R. Wolpaw "EMG contamination of EEG: spectral and topographical characteristics" Clinical Neurophysiology 114 (2003) 1580-1593.

[6] Sparkfun, "Xbee manual" [online] Available: https://www.sparkfun.com/datasheets/Wireless/Zigbee/X Bee-Manual.pdf

[7] Cytron Technologies, "SKXbee starter kit" [online] Available:

http://www.cytron.com.my/datasheet/WirelessDevice/SK Xbee_User's_Manual_v1.pdf

[8] C. Fonseca, J. P. S. Cunha, R.E. Martins, V. M. Ferreira, J. P. M. de Sa, M. A. Barbosa, and A. M. da Silva, "A novel dry active electrode for EEG recording," IEEE Trans. Biomed. Eng., vol. 54, no. 1, pp. 162-165, Jan. 2007.

[9] F. Popescu, Y. Fazli, S. Badower, B. Blankertz, and K.R. Muller," "Single trial classification of motor imagination using 6 dry EEG electrodesm," PLOS ONE, vol. 2 , no. 7, 2007.

[10] A. Taheri, R. Knight, and R. Smith, "A dry electrode for EEG recording", Electroencephalogr. Clin. Neurophysiol. vol. 90, no. 5, pp. 376-383,May 1994.

[11] A. Searle and L. Kirkup, "A direct comparison of wet, dry and insulating bioelectric recording electrodes," Physiol. Meas., vol. 21, no. 2, pp. 71-83, May 2000.

[12] G. Gargiulo, P. Bifulco, R. A. Calvo, M. Cesarelli, C. Jin, A. McEwan, and A. van Schaik, Intelligent and Biosensors. Rijeka, Croatia: In Tech, Jan. 2010, ch. 6.

[13] E. W. Sellers, P. Turner, W. A. Sarnacki, T. Mcmanus, T. M. Vaughan, and B. Matthews, "A novel dry electrode for brain-computer interface," in Proc. 13th Int. Conf. Human-Computer Interac., San Diego, CA, 2009, pp. 623-631.

[14] T. J. Sullivan, S. R. Deiss, T. P. Jung, and G. Cauwenberghs, "A brain- machine interface using drycontact low-noise EEG sensors," in Proc.Conf. Rec. 2008 IEEE Int. Symp. Circuits Syst., Seattle, WA, May 2008,pp. 1986-1989.

[15] J.d.R.Millan, R.Rupp, G.R.Muller-Putz, R. MurraySmith, C.Giugliemma, M.angermann, C. Vidaurre, F.Cincotti, A.Kubler, R. Leeb, C.Neuper, K.-R. Muller, and D.Mattia, "Combining brain-computer interfaces and assistive technologies state-of-the-art and challenges," Frontiers Neurosci., vol. 4, pp. 1-15, 2010.

[16] Neurosky, "Mind-set instruction manual", [online] Available:

http://developer.neurosky.com/docs/doku.php?id=mindse t_instruction_manual. 